\title{
As Artes na Educação Médica: Revisão Sistemática da Literatura
}

\section{Arts in Medical Education: a Systematic Literature Review}

\author{
Lúcia Trindade da Silva Mairot ${ }^{I}$ \\ Beatriz Biasi Gin da Costa ${ }^{I}$ \\ Thaís Pedrosa Moraes Heringer ${ }^{1}$ \\ Raquel Camargos Borges ${ }^{I I}$ \\ Eliane Perlatto Moura ${ }^{\text {II }}$
}

\section{PALAVRAS-CHAVE}

- Educação médica.

- Artes.

- Currículo.
${ }^{I}$ Universidade Federal de São João Del Rey, São João Del Rey, Minas Gerais, Brasil.

${ }^{I I}$ Universidade José do Rosário Vellano, Belo Horizonte, Minas Gerais, Brasil.
Introdução: A introdução das artes no currículo médico tem despertado interesse crescente, uma vez que estas apresentam qualidades únicas que podem auxiliar no desenvolvimento dos aspectos sociais da prática médica, oferecendo maneiras novas e distintas de exploração do conhecimento e da identidade profissional. Objetivo: Avaliar a eficácia da utilização das artes no currículo médico por meio de uma revisão bibliográfica. Metodologia: As bases de dados Lilacs, SciELO, PubMed e Eric foram pesquisadas para artigos publicados sobre estudos que tentaram avaliar a eficácia de uma abordagem baseada em artes na educação médica de graduação. Outros artigos foram identificados por meio de busca ativa. Foram utilizados os seguintes descritores (art or visual arts or paintings or literature or narrative or poetry or theatre or movies or films or cinema) AND (medical education or medical student or medical curriculum). Foram incluídos somente os artigos cujo estudo foi realizado com estudantes de Medicina e cuja eficácia da intervenção foi avaliada por comparação entre grupos (estudos quantitativos) ou pela satisfação do estudante de Medicina por questionário (estudos qualitativos). Os artigos selecionados foram lidos na integra por dois pesquisadores, de modo a identificar o tipo de arte utilizada na intervenção, o autor, a amostra, a metodologia e a conclusão sobre a atividade relatada. Resultados: Foram incluídos 28 artigos no estudo, distribuídos de acordo com o tipo de arte utilizada: $(n=16)$ artes visuais; $(n=6)$ literatura; $(n=3)$ teatro; $(n=3)$ cinema. As competências educacionais sensiveis às artes relatadas nos estudos avaliados foram: habilidades de observação diagnóstica, trabalho em equipe, reflexão e argumentação; facilitar o aprendizado cognitivo; aspectos humanísticos da medicina (empatia/relação médico-paciente); profissionalismo. Alguns estudos afirmam que as intervenções baseadas em artes são eficazes na alteração de atitudes, entretanto não definiram como esse sucesso foi medido. Nenhum estudo considera os efeitos sobre o comportamento. As evidências para o uso de intervenções baseadas em artes para promover habilidades de observação diagnóstica mostraram ser mais fortes. No entanto, seu efeito em outras habilidades clínicas não foi estudado. Conclusão: A arte pode ser uma estratégia facilitadora do aprendizado, uma vez que auxilia o estudante a lidar com a complexidade do ser humano e da saúde humana. Este conhecimento mais amplo sobre a saúde e a doença pode levar à melhoria da relação médico-paciente na prática clínica. Entretanto, devido à natureza qualitativa da maioria dos estudos, baseados principalmente na opinião do estudante sobre as modificações ocorridas em suas atitudes, a eficácia das intervenções nem sempre foi efetivamente demonstrada. 


\section{KEY-WORDS}

- Medical education.

- Arts

- Curriculum.

\begin{abstract}
Introduction: The introduction of the arts into the medical curriculum has sparked increasing interest since they present unique qualities that can aid in the development of the social aspects of medical practice, offering new and distinctive ways of exploring knowledge and professional identity. Objective: To evaluate the effectiveness of the use of the arts in the medical curriculum, through a bibliographical review. Methodology: The Lilacs, Scielo, PubMed and Eric databases were searched for published articles on studies that attempt to evaluate the effectiveness of an arts-based approach in undergraduate medical education. Other articles were identified through active searching. The following descriptors (art or visual arts or paintings or literature or narrative or poetry or theatre or movies or films or cinema) AND (medical education or medical student or medical curriculum) were used. We included only articles related to studies that were conducted with medical students and which evaluated the effectiveness of the intervention by comparison between groups (quantitative studies) or by medical student satisfaction by questionnaire (qualitative studies). The selected articles were read in full in order to identify the type of art used in the intervention, the author, the sample, the methodology and the conclusion about the reported activity. Results: A total of 28 articles were included in the study, distributed according to the type of art used: $(n=16)$ visual arts; $(n=6)$ literature; $(n=3)$ theater; $(n=$ 3) cinema. The arts-sensitive educational skills reported in the studies evaluated were: skills diagnostic observation, teamwork, reflection and argumentation; facilitating cognitive learning; humanistic aspects of medicine (empathy / patient medical relationship); professionalism. Some studies state that arts-based interventions are effective at changing attitudes; however, they did not define how this success was measured. No study considers the effects on behavior. Evidence for the use of arts-based interventions to promote diagnostic observation skills has been shown to be stronger. However, its effect on other clinical skills has not been studied. Conclusion: Art can represent a strategy to facilitate learning, since it helps the student to deal with human complexity and health. This broader understanding of health and illness can lead to improved physician-patient relationships in clinical practice. However, due to the qualitative nature of most of the studies, based mainly on the student's opinion of the changes in their attitudes, the effectiveness of the interventions has not always been effectively demonstrated.
\end{abstract}

Recebido em: 29/11/18

Aceito em: 1/4/19

\section{INTRODUÇÃO}

A arte tem sido proposta como instrumento fundamental de educação, ocupando historicamente papéis diversos desde Platão, que a considerava como base de toda a educação natural. $\mathrm{O}$ envolvimento com as artes e o aprendizado por meio das artes aumenta a exposição individual a múltiplas formas de experimentar o mundo e, ao fazê-lo, aumenta o potencial de desenvolvimento humano ${ }^{1,2}$. Os defensores da utilização das artes na educação citam estudos que mostram que as artes têm valor não só para o objetivo educacional maior de melhorar a aprendizagem dos alunos, mas também para o desenvolvimento cognitivo e neurológico ${ }^{1}$.

Considerando o argumento de que as artes podem melhorar as habilidades de pensamento geral do aluno e as disposições de aprendizagem - persistência na tarefa, atenção mais sustentada, habilidades mais nítidas em observação, ferramentas mais ricas para a expressão e melhor interação social -, muitos pesquisadores apoiam o uso das artes como ferramenta de ensino em domínios não artísticos ${ }^{1}$, como, por exemplo, na educação médica.

A introdução das artes no currículo médico tem despertado interesse crescente e tem sido objeto de vários estudos. $\mathrm{O}$ objetivo da inclusão das artes na educação médica tem variado muito, e as atividades envolvem tanto a visualização como a criação de artes ${ }^{3,4,5}$. Os possíveis resultados da utilização das artes na educação médica incluem uma visão enriquecida da aprendizagem e do desenvolvimento profissional, estimulando o desenvolvimento de várias competências ${ }^{6,7,8}$. Muitos autores descreveram como o treinamento em artes e humanidades podem promover profissionalismo, habilidades de escuta, sensibilidade cultural, ética, empatia ou um compromisso com o humanismo 9 . A arte solicita a visão, a escuta e os demais sentidos como portas de entrada para uma compreensão mais significativa da relação médico-paciente ${ }^{10}$. 
A medicina é descrita como ciência e arte, sendo a ciência focada no conhecimento objetivo, técnico (competência ou cura), e os elementos artísticos focados no lado humano (empatia ou cuidado) ${ }^{11}$. Ambas as abordagens da realidade (arte e ciência) devem ser integradas de modo a melhorar o processo de educação médica. Isso pode ser implementado mediante uma análise de obras-primas artísticas universais (literatura, poesia, pintura, etc.) que lidam com tópicos humanísticos decisivos, que servem de fonte de inspiração (fator desencadeante) para a geração de ideias (hipótese) que podem esclarecer os fenômenos observados tanto nos níveis de saúde como nos níveis experimentais ${ }^{78,12}$.

A Arte apresenta uma ambiguidade com muitas camadas de significados incorporados e sua interpretação exige o envolvimento de sensibilidade, imaginação e reflexão. Promover essas habilidades é um grande objetivo de aprendizagem, porque são essenciais à competência clínica e ao desenvolvimento profissional $^{5}$.

Neste contexto, a inserção da arte como ferramenta educacional para treinamento profissional, dada sua característica simbólica, é ideal para transmitir os aspectos da humanidade (medo da morte, poder terapêutico da empatia, etc.) bem como operar como um gatilho para o conhecimento do mundo em um nível inconsciente (intuição). Assim, a educação médica passa a incorporar o desafio de se adequar, para oferecer aos médicos produtos finais de seus currículos uma formação humanista e humanizadora ${ }^{13}$.

Diante do interesse crescente da inclusão das artes na educação médica, buscou-se analisar, por meio de uma revisão sistemática da literatura, a eficácia da utilização das artes no ensino médico.

\section{METODOLOGIA}

Trata-se de um estudo de revisão sistemática, conduzido conforme recomendações da Cochrane Collaboration ${ }^{14}$.

\section{Definições conceituais e critérios de elegibilidade}

A pergunta deste estudo seguiu a estratégia Pico, que representa um acrônimo para População, Intervenção, Comparação e Desfecho ${ }^{15}$. Os principais termos usados nesta revisão foram definidos da seguinte forma: $\mathrm{P}$ = estudantes de Medicina; $\mathrm{I}=$ utilização das artes como estratégia educacional no currículo de Medicina; $\mathrm{C}$ = grupo intervenção e grupo controle ou antes e depois; $\mathrm{O}=$ eficácia da estratégia.

\section{Estratégias de busca}

Para identificar as publicações que compuseram este estudo, realizou-se busca online nas bases de dados eletrônicas Lilacs, Scielo, Pubmed e Eric. Os descritores utilizados para direcio- nar a pesquisa foram: (Art or visual arts or paintings or narrative or poetry or theatre or movies or cinema) AND (Medical education or medical students or medical curriculum) (Quadro 1). Buscas manuais foram feitas nas referências bibliográficas dos artigos encontrados.

\begin{tabular}{|c|c|}
\hline & $\begin{array}{c}\text { QUADRO } 1 \\
\text { Estratégia de busca utilizada para a } \\
\text { pesquisa nas bases de dados }\end{array}$ \\
\hline $\begin{array}{l}\text { Número da } \\
\text { estratégia }\end{array}$ & Descritores \\
\hline 1 & $\begin{array}{l}\text { Arts OR visual arts OR painting OR literature OR } \\
\text { narrative OR poetry OR theatre OR movies OR films OR } \\
\text { cinema }\end{array}$ \\
\hline 2 & $\begin{array}{l}\text { Medical education OR medical students OR medical } \\
\text { curriculum }\end{array}$ \\
\hline
\end{tabular}

2. Descritores relativos à população ( $\mathrm{P}$ da estratégia Pico).

Não foi delimitado o ano de publicação para inclusão no estudo. A consulta à base de dados foi realizada de abril de 2016 a setembro de 2017. Os idiomas de redação dos artigos aceitos foram inglês, português, francês e espanhol.

Seleção dos estudos e critérios de inclusão e exclusão Como critérios de inclusão, foram utilizados somente os artigos cujo estudo foi realizado com estudantes de Medicina e cuja eficácia da intervenção foi avaliada por comparação entre grupos (estudos quantitativos) ou pela satisfação dos estudantes (estudos qualitativos). Foram excluídos os artigos que abordavam a utilização da arte em pacientes e profissionais de outras áreas da saúde, bem como os que descreviam a importância da arte ou aqueles que não descreviam a intervenção utilizada.

A avaliação dos títulos e resumos foi realizada por dois pesquisadores, de forma independente, obedecendo rigorosamente aos critérios de inclusão e exclusão definidos no protocolo de pesquisa. Em casos de discordância, os pesquisadores tentaram encontrar concordância em uma reunião de consenso.

\section{Processo de coleta e extração de dados}

Os dados foram extraídos de maneira padronizada e aplicada aos trabalhos pelos dois revisores, que trabalharam independentemente e levaram em consideração as seguintes variáveis: autor, nome do artigo, local, desenho do estudo, amostra, intervenção utilizada, análise dos dados, resultados e conclusão.

Os artigos incluíram duas categorias principais de resultados: quantitativa e qualitativa. Os resultados quantitativos foram avaliados por meio de dados descritivos (frequências, porcentagens, proporções), correlações e variância (t-tests, análise de variância, Anova e outros), e $p<0,05$ foi conside- 
rado de significância. Os resultados qualitativos foram avaliados por meio de análise de conteúdo.

\section{Qualidade dos estudos}

A qualidade dos estudos foi avaliada conforme os seguintes critérios: descrição: (1) da população estudada, (2) da intervenção utilizada, (3) do processo de coleta de dados, (4) do método analítico, (5) dos resultados e (6) conclusão. A cada estudo foi atribuída uma pontuação que variou de 0 a 6 . A qualidade de cada artigo foi classificada como baixa (1-2), média (3-4) ou alta (5-6). Foram considerados aptos a serem incluídos neste estudo os artigos que apresentaram classificação média e alta na análise de qualidade. Os dois revisores chegaram a um consenso sobre a classificação de todos os trabalhos.

Como se trata de uma pesquisa documental, o estudo não foi submetido ao Comitê de Ética em Pesquisa com Seres Humanos.

\section{RESULTADOS}

\section{Seleção dos estudos}

A seleção dos artigos para a revisão sistemática foi feita de acordo com o fluxograma apresentado na Figura 1. Foram en- contrados 3.053 artigos ao se utilizarem os descritores arts and medical education. Houve a inclusão de cinco artigos identificados em referências de artigos e artigos de revisão, totalizando, então, 3.058. Destes, 2.568 artigos foram excluídos após o refinamento da busca utilizando-se os descritores (visual arts, painting, literature, narrative, poetry, theatre, films, cinema, movie AND medical education, medical curriculum, medical students). Foram eliminadas as duplicatas e os estudos realizados com pacientes ou profissionais de outras áreas da saúde. Foram selecionados, então, 490 artigos. Destes, 424 foram excluídos após análise dos resumos por se tratar de artigos de descrição e artigos de opinião. Restaram, então, 66 artigos, cujos textos foram lidos na íntegra. Destes, 38 foram excluídos porque não se encaixavam nos critérios de inclusão do estudo: estudos de relato de experiência $(n=20)$; estudos de revisão $(n=5)$; estudos sem descrição da intervenção realizada $(n=10)$; estudos sem avaliação final da eficácia da estratégia $(n=30)$. Assim, foram selecionados 28 artigos que compuseram a amostra. Os artigos selecionados estão descritos na Figura 1.

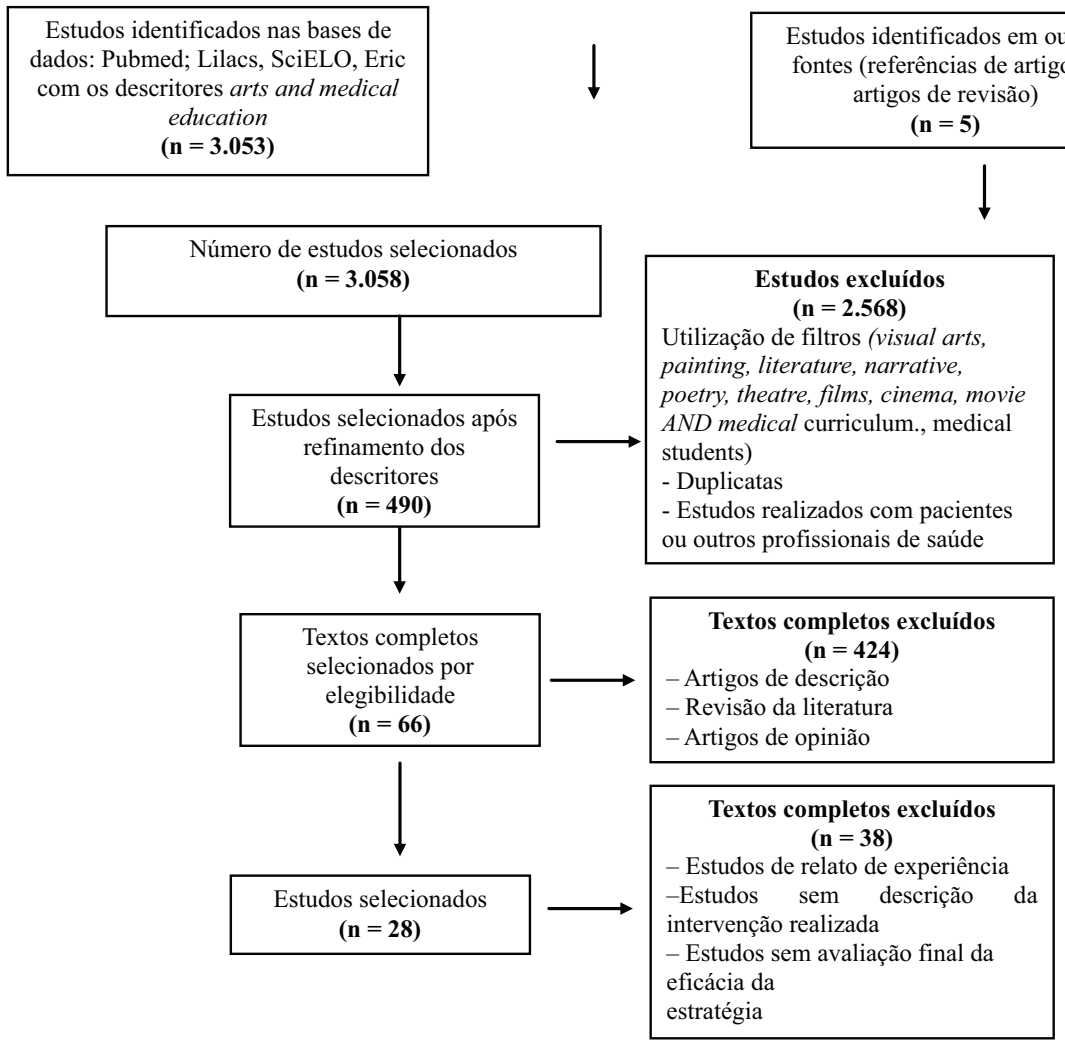




\section{Características dos estudos}

Os estudos identificados nesta revisão foram publicados entre 2001 e 2017. Os primeiros autores dos artigos eram de instituições situadas nos Estados Unidos $(n=16)$, Reino Unido $(n=3)$, Brasil $(n=2)$, Austrália $(n=1)$, Canadá $(n=1)$, Nepal $(n=1)$, Suécia $(n=1)$, Turquia $(n=1)$, Eslovênia $(n=1)$ e Cuba $(n=1)$.

A distribuição dos estudos segundo o tipo de arte utilizada na intervenção foi a seguinte: artes visuais ( $n=16)$; narrativa $(\mathrm{n}=4)$; teatro $(\mathrm{n}=3)$; cinema $(\mathrm{n}=3)$; narrativa/poema $(\mathrm{n}$
=2). Dos 28 artigos selecionados, 13 (46\%) foram estudos qualitativos, 10 (36\%) foram quantitativos e qualitativos e $5(18 \%)$ foram estudos quantitativos. A maioria dos estudos (23-82\%) não utilizou grupo controle; 10 (36\%) coletaram dados pré- e pós-teste; 18 (64\%) coletaram dados somente após a intervenção; e 13 (46\%) tiveram a colaboração de profissionais da arte. O número total de sujeitos abordados no estudo variou de 5 a 217. As intervenções realizadas estão descritas no Quadro 2.

\section{Autor/Título
Artigos
$\begin{aligned} & \text { Dolev et al }{ }^{16} \text {. } \\ & \text { Use of fine Art to Enhance Visual Diagnostic skills } \\ & \text { / EUA }\end{aligned}$}

Pretorius et al ${ }^{17}$

Is it Impressionism or Is It Medicine?/ EUA

\section{Naghshineh et al ${ }^{26}$ \\ Formal Art Observation Training Improves \\ Medical Students' Visual Diagnostic Skills/ EUA \\ Jasani \& Sacks ${ }^{18}$ \\ Utilizing Visual Art to enhance the Clinical \\ Observation Skills of Medical Students /EUA \\ Bell \& Evans ${ }^{19}$ \\ Art, Anatomy and Medicine: Is There a Place for \\ Art in Medical Education?/ Reino Unido \\ Bardes, et al ${ }^{20}$ \\ Learning to look: developing clinical observational \\ skills at an art museum / EUA}

Friedlaender \& Friedlaender ${ }^{22}$

Art in Science: Enhancing Observational skills /

EUA

Braverman $^{28}$

To see or not to see: How visual training can improve observational skills / EUA

Kidd et al ${ }^{27}$

Using visual art an collaborative reflection to

explore medical attitudes toward vulnerable

persons / Canadá

Gurwin et al ${ }^{21}$

A randomized controlled study of art observation

training to improve medical student ophthalmology skills / EUA

Finn et al ${ }^{30}$

The impact of color and role on retention of

knowledge. A body-painting study within

undergraduate medicine / Reino Unido

Tapajós ${ }^{25}$

HIV/AIDS in visual arts: applying discipline-

based art education (DBAE) to medical humanities

/Brasil

\section{QUADRO 2}

Tipo de arte

Artes visuais

Artes visuais

Artes visuai

Artes visuais

Artes visuais

Artes visuais

Artes visuais

Artes visuais

Artes visuais Estudo experimental pré e pós-teste. Quantitativo e qualitativo com estudantes de medicina, pesquisadores e profissionais da saúde $(n=20)$. Palestras relacionando artes visuais e diagnóstico físico seguido de descrição, realizada pelos estudantes, de casos clínicos apresentados.

Artes visuais Estudo experimental randomizado com 36 estudantes do $1^{\circ}$ ano de medicina. Análise de obras de arte em um museu seguido por descrição de características visuais necessárias para o diagnóstico

Artes visuais Estudo experimental randomizado com 117 estudantes do $1^{\circ}$ ano de medicina. Pintura do corpo (utilizando cores e preto e branco).

Artes visuais Estudo experimental qualitativo com 50 estudantes de medicina do $2^{\circ}$ ano ao 6 ano medicina. Curso em módulos: artes gráficas, artes plásticas, fotografia e memorial AIDS. 


\begin{tabular}{|c|c|c|}
\hline Autor/Título & Tipo de arte & Tipo de estudo/Intervenção \\
\hline $\begin{array}{l}\text { Shankar }{ }^{23} \\
\text { Student feedback on the use of paintings in } \\
\text { Sparshanam, the Medical Humanities Module at } \\
\text { Kist Medical College. / Nepal }\end{array}$ & Artes visuais & $\begin{array}{l}\text { Estudo experimental quantitativo e qualitativo com } 100 \text { estudantes do } 1^{\circ} \text { ano } \\
\text { de medicina. Análise de pinturas com reflexão e elaboração de narrativas, } \\
\text { música, poema ou role play. }\end{array}$ \\
\hline $\begin{array}{l}\text { Moore et al } 31 \\
\text { Developing observational skills and knowledge } \\
\text { of anatomical relationship using plastinated } \\
\text { specimens / EUA }\end{array}$ & & $\begin{array}{l}\text { Estudo experimental qualitativo com } 5 \text { estudantes do } 1^{\circ} \text { ano de medicina. } \\
\text { Workshop dividido em } 3 \text { sessões: palestra sobre técnica básica de desenho de } \\
\text { estruturas do corpo; perspectivas e proporções do rosto humano; importantes } \\
\text { artistas anatomistas do Renascimento. }\end{array}$ \\
\hline
\end{tabular}

Student Education / EUA

McMenamin ${ }^{29}$

Body Painting as a Tool in Clinical Anatomy

Teaching / Austrália

Arjmand ${ }^{36}$

The Use of Narrative in Medical Education / EUA

Estudo experimental qualitativo e quantitativo com 217 estudantes de medicina. Pinturas de estruturas anatômicas sobre um modelo vivo (alunos foram encorajados a alternar entre pintura e atuação como modelo).

\section{Miller et al ${ }^{34}$}

Sounding Narrative Medicine: Studying Students

Professional Identity Development at Columbia

University / EUA

Easton ${ }^{35}$

How medical teachers use narratives in lectures: a quality study / Reino Unido

Van Winkler et al ${ }^{37}$

Use of poems Written by Physicians to Elicit

Reflection by Students in a Medical Biochemistry

Course / EUA

\section{Shapiro et al ${ }^{32}$}

Teaching Empaty to First year Medical Students:

Evaluation of an Elective Literature and Medicine Course / EUA

Jacobson et al ${ }^{33}$

A literature and medicine special study module run by academics in general practice: two evaluation and the lessons learnt / Reino Unido

Reilly et al $^{36}$

Using Theater to Increase Empathy Training in Medical Students / EUA

Ünalan et al ${ }^{39}$

Using theatre in education a traditional lecture oriented medical curriculum / Turquia

Jacobsen et al ${ }^{40}$

Analysis of role-play in medical communication

training using a theatrical device the fourth wall / Suécia

Klemenc-Ketis \& Kersnik ${ }^{43}$

Using movies to teach professionalism to medical students / Eslovênia

\section{Landsberg ${ }^{42}$}

Vendo o outro através da tela: cinema,

humanização da educação / Brasil

Figaredo \& Garcia ${ }^{41}$

El cine como herramienta em la docencia de

Psiquiatria / Cuba
Narrativa

Estudo qualitativo com 5 estudantes do $4^{\circ}$ ano de medicina. Curso para aprimorar habilidades narrativas sobre temas ligados à comunicação médicopaciente.

Narrativa Estudo qualitativo com 130 estudantes do $2^{\circ}$ ano de medicina. Seminários sobre narrativa (textos relacionados com medicina intensiva) seguidos de discussão e transcrição de experiências.

Narrativa

Estudo experimental qualitativo com estudantes do $1^{\circ}$ ano de medicina. Narrativas baseadas na transcrição das gravações de áudio de palestras médicas.

Poemas

Estudo experimental qualitativo com 196 estudantes do $1^{\circ}$ ano de medicina. Associação entre elementos presentes em poemas e valores humanísticos e discussão sobre dissonâncias de opiniões em grupo.

Narrativas e Estudo experimental randomizado com pré e pós teste. Qualitativo e poemas quantitativo com 16 estudantes do $1^{\circ}$ ano de medicina. Seminários sobre narrativa seguidos de transcrição de experiências.

Narrativa

Estudo experimental qualitativo com 8 estudantes do $3^{\circ}$ ano de medicina. Seminários sobre narrativa seguidos de discussão e transcrição.

Teatro

Estudo experimental qualitativo com 167 estudantes do $1^{\circ}$ ano de medicina. Workshop de teatro (uso de jogos de teatro, fotografia e escrita reflexiva) para construir empatia/autorreflexão.

Teatro Estudo experimental qualitativo e quantitativo com 49 estudantes de medicina. Curso preparatório de 2 semanas para escrever diferentes cenários dramatizados sobre tema de medicina, desempenho teatral seguido de discussão.

Teatro Estudo experimental qualitativo com 30 estudantes do último ano de medicina Workshop filmado com a utilização de role-play e técnica conhecida como a "quarta parede", seguida da análise do material filmado.

Cinema Estudo experimental qualitativo com 11 estudantes do $4^{\circ}$ ano de medicina. Curso de 4 meses sobre profissionalismo com exibição de filmes sobre temas escolhidos pelos estudantes, seguidos de discussão e apresentação escrita e oral.

Cinema Estudo experimental qualitativo e quantitativo com 34 estudantes do $1^{\circ}$ e $6^{\circ}$ período de medicina. Projeção de filmes seguido de discussão multidisciplinar com presença de psicanalista e profissional do cinema.

Cinema Estudo qualitativo com 43 estudantes do $5^{\circ}$ ano de medicina. Projeção de filmes (1vez por semana) relacionado ao conteúdo do programa da disciplina de Psiquiatria, após palestra introdutória e antes do seminário correspondente. 


\section{Competências educacionais sensíveis às artes visuais}

A observação detalhada de pinturas, seguida de discussão e ou reflexão, foi capaz de desenvolver a capacidade de observação clínica dos estudantes, com possíveis consequências positivas na relação médico-paciente ${ }^{16-21}$. A experiência de análise visual de uma pintura e a narrativa que articula o que se vê pela primeira vez sem interpretação podem ser uma ferramenta importante no aprimoramento das habilidades de observação ${ }^{22}$. Shankar et al..$^{23}$ discutem em seu estudo como a utilização de pinturas na educação médica pode libertar a imaginação, auxiliar os alunos a ver novas perspectivas e pensar de forma crítica e criativa sobre os problemas que podem encontrar em sua futura carreira. Elder et al. ${ }^{24}$ e Tapajós ${ }^{25}$ discutem como as artes visuais podem melhorar as habilidades de observação, descrição, interpretação e resposta dos estudantes de Medicina em relação aos aspectos subjetivos do cuidado médico. Naghshineh et al. ${ }^{26}$ e Kidd et al. ${ }^{27}$ relatam que relacionar as pinturas com o diagnóstico físico foi capaz de melhorar a acurácia diagnóstica dos estudantes.

As artes visuais desencadeiam sentimentos e transmitem consciência de valores e sentimentos pessoais ${ }^{16}$. Segundo Braverman $^{28}$, a arte possibilita que o aluno aprenda a aceitar e a considerar interpretações alternativas, além de estimular o trabalho em equipe, que é essencial na prática profissional. As artes promovem habilidades específicas e psicomotoras úteis à profissão, como a visão espacial e a capacidade de pensar em três dimensões. Segundo Mcmenamin ${ }^{29}$, o uso da pintura corporal para auxiliar o aprendizado dos alunos sobre aspectos da anatomia clinicamente relevantes provou ser uma estratégia educacional positiva na aprendizagem da topografia humana. Finn et al. ${ }^{30}$ analisam o impacto das cores na pintura corporal e acreditam que exista uma associação entre retenção de conhecimentos e utilização de cores. Moore et al. ${ }^{31}$ discutem como uma oficina de arte e anatomia pode auxiliar os estudantes a melhorar a capacidade de detectar detalhes e levar os alunos a uma reflexão sobre os aspectos humanísticos da anatomia. Além disso, as artes visuais são divertidas e recreativas, na medida em que geram prazer e permitem um relaxamento do estresse diário ${ }^{29}$.

\section{Competências educacionais sensíveis à utilização da literatura}

Shapiro et al. ${ }^{32}$ discutem como o uso da literatura (leitura de poesia, paródias, notícias sobre o relacionamento médico-paciente) pode melhorar a empatia e interesse pela literatura dos alunos ao estimular um olhar mais amplo sobre a relação médico-paciente. Jacobson et al..$^{33}$ sustentam que a literatura pode ser utilizada para auxiliar o aluno a lidar com a com- plexidade do adoecer, que envolve sentimentos (do paciente e do médico), atitudes e comportamentos. Miller et al. ${ }^{34}$ e Easton $^{35}$ observam que a narrativa pode ser útil à aprendizagem na medicina, uma vez que, ao abordar capacidades complexas interiores, interpessoais, perceptivas e expressivas, pode ser particularmente relevante na promoção de aspectos humanísticos da medicina, incluindo a identidade profissional e empatia. Arjmand ${ }^{36}$ relata que o exercício de relacionar os textos literários com a prática clínica, no treinamento de habilidades, estimula o estudante a avaliar sua habilidade de compreender e interpretar a fala do paciente durante a prática clínica. Van Winkle et al. ${ }^{37}$ demonstram que a análise dos poemas estimulou a reflexão crítica dos estudantes sobre valores humanísticos e a prática profissional, bem como a lidar com a dissonância de opiniões, estimulando maior interação no trabalho em equipe.

\section{Competências educacionais sensíveis à utilização do teatro}

A empatia tem sido reconhecida como um elemento essencial na educação médica. Reilly et al. ${ }^{38}$ relatam que o teatro pode ser utilizado como uma ferramenta eficiente para o treinamento da habilidade de demonstrar empatia em estudantes de Medicina. Ünalan et al. ${ }^{39}$ reconhecem a utilização do teatro na educação médica como uma ferramenta para facilitar o ensino não só de habilidades de comunicação e narrativa de história, mas também de problemas clínicos comuns.

Jacobsen et al. ${ }^{40}$ discutem que o conceito teatral "a quarta parede" - onde todos interagem: atores, estudantes, moderadores e plateia - pode ser uma ferramenta útil para treinar habilidades de comunicação em cenário simulado.

\section{Competências educacionais sensíveis à utilização de cinema e filmes}

A constatação de que o cinema é, provavelmente, o tipo de arte com maior popularidade entre os alunos confirma o seu potencial como recurso didático humanizador. Neste contexto, Figaredo e Garcia ${ }^{41}$ relatam a experiência positiva da utilização do cinema como ferramenta útil no ensino de Psiquiatria para estudantes de Medicina e Landsberg ${ }^{42}$ discute os aspectos positivos da correlação dos filmes com a prática médica. Klemenic-Ketis e Kersnik ${ }^{43}$ usam filmes para ensinar profissionalismo a estudantes de Medicina. Os autores relatam que por meio dos filmes os alunos podem explorar seus valores, crenças e atitudes em relação às características do profissionalismo sem sentir que sua integridade pessoal tenha sido ameaçada. 


\section{DISCUSSÃO}

Esta revisão sugere que a utilização das artes no currículo médico pode ser uma ferramenta útil ao desenvolvimento dos aspectos mais subjetivos relacionados à prática clínica. De La Croix et al. ${ }^{44}$ relatam que os programas de ensino baseados em artes muitas vezes são incluídos nos currículos das escolas médicas sob o guarda-chuva de humanidades médicas. Nossa análise revelou que as intervenções baseadas em artes, dos estudos selecionados, além de abordarem aspectos humanísticos da medicina, versaram também sobre o desenvolvimento de observação diagnóstica, o aprendizado cognitivo, a habilidade de reflexão e argumentação, o profissionalismo e a capacidade de trabalhar em equipe. Estes resultados demonstram o amplo espectro de abordagem que é sensível à utilização da arte como estratégia educacional. Segundo Haidet $e t$ al. ${ }^{4}$, a natureza metafórica e representacional das artes, bem como sua subjetividade, qualidades ambíguas e complexas e universalidade são características que podem promover ricas experiências de aprendizagem. Estas características auxiliam a desafiar o pensamento concreto ou literal e também servem para legitimar as experiências e emoções pessoais dos alunos em lidar com uma variedade de tópicos. As artes, portanto, podem fornecer subsídios para repensar a educação médica e a prática médica.

Entretanto, nossa análise destacou alguns pontos relevantes nos estudos avaliados. Observamos que todos os artigos incluídos neste estudo relataram que suas intervenções foram eficazes em promover modificações positivas nos estudantes, mas somente $29 \%$ deles definiram como esse sucesso foi medido. Essa falta de clareza apareceu principalmente na descrição de como as intervenções baseadas em artes poderiam modificar habilidades e atitudes e de como elas efetivamente o fizeram.

Outro fato observado foi a falta de padronização do método de avaliação utilizado para medir a eficácia da intervenção. Observou-se que a grande maioria dos estudos utilizou questionários desenvolvidos pelos próprios pesquisadores para avaliar a percepção dos estudantes sobre a intervenção realizada, o que dificulta a comparação da intervenção com outros estudos. Somente alguns estudos relatam a utilização de perguntas baseadas no método de ensino denominado Estratégias de Pensamento Visual (Visual Thinging Strategies VTS), desenvolvido por Housen ${ }^{45}$, que vem sendo utilizado com sucesso na educação médica, pois estimula a discussão de ideias em grupo e a habilidade da escuta atenta, com implicações na prática médica ${ }^{46}$. Este método foi desenvolvido para facilitar as discussões sobre obras de arte, promovendo habilidades de pensamento mais amplas e interações colaborativas entre pares. Ele se baseia em três perguntas sequenciais feitas por facilitadores: O que está acontecendo nesta imagem? O que você vê na imagem que leva você a dizer isto? O que mais você pode encontrar? ${ }^{45}$.

Vários estudos basearam o sucesso da intervenção na própria opinião dos estudantes sobre a mudança de suas atitudes. Isto deve ser considerado uma limitação do estudo, uma vez que mudança de atitude não se traduz necessariamente em mudança de comportamento.

Observou-se também que a avaliação da aprendizagem, nos estudos selecionados, utilizou, na maioria dos casos, processos de curto prazo (logo após a intervenção), tendo como objetivo avaliar o desenvolvimento de autoconsciência, abertura para outras perspectivas e maior habilidade para lidar com ambiguidades. Entretanto, nestes casos, deve-se levar em consideração que observações avaliadas imediatamente após a intervenção não permitem identificar o impacto de longo prazo da estratégia adotada. Naghshineh et al. ${ }^{26}$ relatam a importância de uma avaliação longitudinal na avaliação da eficácia da utilização das artes no currículo médico.

Nos estudos que utilizaram o processo de avaliação de longo prazo, o objetivo principal foi avaliar a opinião do estudante sobre a influência da intervenção na prática clínica, não havendo, portanto, uma avaliação efetiva da eficácia da intervenção na mudança de comportamento. Perry et al..$^{7}$ encontraram resultados semelhantes e sugeriram a necessidade de estudos adicionais para avaliar intervenções baseadas em artes em termos de seus efeitos nas atitudes e no comportamento.

Outro fato que merece ser destacado nos estudos selecionados é a ausência de utilização de um grupo controle na grande maioria dos estudos ( $82 \%)$. A utilização de um só grupo de estudo pode levar a um viés de atitudes que podem dominar os membros do grupo ${ }^{7}$.

Também se observou que $46 \%$ dos estudos envolveram a colaboração de artistas ou educadores de artes, levantando questões sobre a importância da experiência necessária e da especialização nas artes para projetar e desenvolver tais intervenções. Osman et al. ${ }^{47}$ sugerem em seu estudo que a colaboração de artistas e educadores de artes no projeto de intervenções é vital para trazer clareza para o papel das intervenções. Segundo esses autores, artistas e educadores de artes podem identificar comportamentos ou habilidades específicas que as intervenções baseadas em artes podem oferecer à educação na área da saúde. Como o conhecimento é inevitavelmente moldado pela experiência disciplinar, a visão dos artistas pode auxiliar na identificação de pontos relevantes que podem não ser percebidos pelos educadores da saúde.

Como fatores limitantes da utilização das artes como estratégia educacional, deve-se destacar o desconforto e a ansie- 
dade apresentados por alguns estudantes ao participarem de atividades baseadas em arte. Deve-se também levar em conta que alguns estudantes não veem a relevância da utilização da arte no currículo ${ }^{5}$.

Assim, os estudos sugerem que os estudantes, no geral, referem satisfação com as estratégias baseadas em arte no currículo médico. Entretanto, devido à natureza subjetiva da maioria dos estudos, baseados principalmente na opinião do estudante sobre as modificações ocorridas em suas atitudes, a eficácia das intervenções nem sempre foi efetivamente demonstrada. Mais estudos de metodologias mistas (qualitativos e quantitativos) e com características longitudinais são necessários para evidenciar o verdadeiro efeito das artes como estratégia educacional no currículo médico.

\section{CONCLUSÃO}

Com base nos resultados dos estudos, podemos concluir que o aprendizado por meio das artes pode fornecer aos estudantes de Medicina habilidades para fortalecer suas práticas, se comunicar de forma mais efetiva e melhorar sua confiança. Além disso, a arte pode ser uma experiência de desenvolvimento pessoal e profissional e representa uma fonte de percepção das experiências dos pacientes e do contexto social, cultural e histórico das práticas médicas.

Entretanto, devido à natureza subjetiva das artes e à ausência de instrumentos padronizados para avaliação das intervenções, sua efetividade como estratégia educacional ainda precisa ser provada.

\section{REFERÊNCIAS}

1. Hardiman M. Education and the Arts: Education Every Child in the Spirit of Inquiry and Joy. Creative Education [online]. 2016; 7: [Capturado 20 set. 2017]; 1913-28. Disponível em: http://www.scirp.org/JJournal/PaperInformation.aspx?PaperID=70138

2. Aprotosoaie-Iftimi AM. The role of art in human development. George Enescu University of Arts. Iasi of Romania 2015;9(10):292-98.

3. Kumagai, AK. Perspective: Acts of interpretation a philosophical approach to using arts in medical education. Acad Med. 2012; 87(8): 1138-44.

4. Haidet P, Jarecke J, Adams NE, Stuckey HL, Green MJ, Shapiro D et al. A guiding framework to maximise the power of the arts in medical education: a systematic review an metasynthesis. Med Educ 2016;50(30):320-31.

5. Frich JC, Fugelli P. Medicine and the arts in the undergraduate medical curriculum at the University of Oslo, Faculty of Medicine, Oslo, Norway. Acad Med 2003;78(10):1036-38.
6. Katz J, Khosshbin S. Can visual arts training improve physician performance? Trans Am Clin Climatol Assoc 2014;125:331-42.

7. Perry M, Maffulli N, Willson S, Morrissey D. The effectiveness of arts-based interventions in medical education: a literature review. Med Educ 2011;45(2):141-48.

8. Lake J, Jackson L, Hardman CA. A fresh perspective on medical education the lens of the arts. Med Educ 2015;49(8):759-72.

9. Jones DS. A complete medical education includes the arts and humanities. Virtual Mentor 2014; 16(8):636-41.

10. Heyn R, Familiari G. Visual thinking strategy in the medical curriculum: training the "clinical eye" in classroom and art museums. Ital J Anat Embryol 2015;120(S1):235.

11. Lazarus PA, Rosslyn FM. The Arts in Medicine: setting up and evaluation a new special study module at Leicester Warwick Medical School. Med Educ 2003;37(6):553-59.

12. Musso C, Enz P. Art as an education tool in medicine. Arch Argent Pediatr 2014;112(6):494-95.

13. Binz MC, Menezes Filho EW, Jatene FB. Novas tendências, velhas atitudes: as distâncias entre valores humanísticos e inter-relações observadas em um espaço docente e assistencial. Rev Bras Educ Med 2010;34(1):28-42.

14. Higgins JPT, Green S (editors). Cochrane Handbook for Systematic Reviews ofInterventions Version 5.1.0 [updatedMarch 2011]. The Cochrane Collaboration, 2011. Available from http:// handbook.cochrane.org.

15. Santos CMC; Pimenta CAM; Nobre MRC. A estratégia PICO para a construção da pergunta de pesquisa e busca de evidências. Rev Latino Am Enfermagem, 2007;15(3):508511.

16. Dolev JC, Friedlander LK, Braverman I. Use of fine art to enhance visual diagnostic skills. JAMA 2001;286(9):102021.

17. Pretorius R, Lohr GO, Mor N, Zinnerstrom KH, Blo$\mathrm{hm}$ AT. Is it impressionism or is medicine? Fam Med 2009;41(4):246-48.

18. Jasani S, Saks N. Utilizing visual art to enhance the clinical observation skills of medical students. Med Teach 2013;35(7):1327-31

19. Bell L, Evans D. Art, anatomy and medicine: is there a place for art in medical education. Anat Sci Educ 2014;7(5):37078.

20. Bardes CL, Gilliers D, Herman A. Learning to look: developing observational skills at an art museum. Med Educ 2001;35(12):1157-61.

21. Gurwin J, Revere KE, Niepold S, Bassett B, Mitchell R, Davidson $S$ et al. A randomized controlled study of art obser- 
vation training to improve medical student ophthalmology skills. Ophthalmology 2018;125(1):8-14.

22. Friedlaender G, Friedlaender L. Art in Science: Enhancing observational skills. Clin Orthop Relat Res 2013;471(7):2065-67.

23. Shankar R, Piryani R, Upadhyay-Dhungel K. Student feedback on the use of paintings in Sparshanam, the Medical Humanities module at KIST Medical College. BMC Med Educ 2011(8):11-19.

24. Elder N, Tobias B, Lucero-Criswell A, Goldenhar L. The art of observation: impact of a family medicine and art museum partnership on student education. Fam Med 2006;38(6):393-98.

25. Tapajós R. HIV/AIDS in the visual arts: applying discipline-based art education (DBAE) to medical humanities. Med Educ 2003;37(6):563-70.

26. Naghshineh S, Hafler JP, Miller AR, Blanco MA, Lipsitz SR, Dubroff RP et al. Formal art observation training improves medical students visual diagnostic skills. J Gen Intern Med 2008; 23(7):991-97.

27. Kidd M, Nixon L, Rosenal T, Jackson R, Pereles L, Mitchell I et al. Using visual art and collaborative reflection to explore medical attitude toward vulnerable persons. Can Med Educ J 2016;7(1):22-30.

28. Braverman I. To see or not to see: how visual training can improve observational skills. Clin Dermatol 2011;29(3):34346.

29. McMenamin PG. Body painting as a tool in clinical anatomy teaching. Anat Sci Educ 2008;1(4): 139-44.

30. Finn G, White P, Abdelbagi I. The impact of color and role on retention of knowledge: a body painting study within undergraduate medicine. Anat Sci Educ 2011;4(6):311-17.

31. Moore C, Lowe C, Lawrence J, Borchers P. Developing observational skills and knowledge of anatomical relationships in an art and anatomy workshop using plastinated specimens. Anat Sci Educ 2011;4(5):294-301.

32. Shapiro J, Morrison E, Boker J. Teaching empathy to first year medical students: evaluation of an effective literature and medicine course. Educ Health 2004;17(1):73-84.

33. Jacobson L, Grant A, Hood K, Lewis W, Robling M, Prout $\mathrm{H}$ et al. A literature and medicine special study module run by academics in general practice; two evaluations and the lessons learnt. Med Humanit.2004;30(2):98-100.

34. Miller E, Balmer D, Hermann N, Graham G, Charon R. Sounding narrative medicine: studying student's professional identity development at Columbia University College of physicians and surgeons. Acad Med 2014;89(2):33542.
35. Easton G. How medical teachers use narratives in lectures: a quality study. BMC Med Educ 2016;16(3):1-12.

36. Arjmand S. The use of narrative in medical education. Journal for Learning through the Arts . Journal for Learning through the Arts, [Internet],2012. 8. (1), [Capturado 15 mar. 2017]. Disponível em: <http://escholarship.org/ uc/item/1b95d8vv\#page-3, https://doi.org/10.21977/ D9812656

37. Van Winkle L, Robson C, Chandar N, Green JM. Use of poems written by physicians to elicit cfriticalreflection by students in a medical biochemistry course. Journal for Learning through the Arts. 2011.7(1). [Capturado 10 set. 2017]. Disponível em http://escholarship.org/uc/ item/7513c5mv, https:/ / doi.org/10.21977/D9812656

38. Reilly J, Trial J, Piver DE, Schaff PB. Using theater to increase empathy training in medical education. Journal for Learning through the Arts 2012, 8(1). [Capturado 20 ago. 2007]. Disponível em: https:/ / escholarship.org/uc/ item/68x7949, https:/ / doi.org/10.21977/D9812646

39. Ünalan P, Uzuner A, Çifçili S, Akman M, Hancıoğlu S, Thulesius HO. Using theatre in education a traditional lecture oriented medical curriculum. BMC Med Educ 2009, 9:73 [Capturado 10 ago. 2007]. Disponível em: https:// www.ncbi.nlm.nih.gov/pmc/articles / PMC2803161, doi: 10.1186/1472-6920-9-73

40. Jacobsen I, Baerheim A, Lepp MR, Schei E. Analysis of role-play in medical communication training using a theatrical device the fourth wall. BMC Med Educ 2006;6:51.

41. Figaredo PH, Garcia FP. El cine como herramienta em la docencia de Psiquiatria. Rev Human Med 2013;13(1):24465.

42. Landsberg G. Vendo o outro através da tela: cinema, humanização da educação médica e Medicina de Família e Comunidade. RBMFC 2009;4(16):298-304.

43. Klemenc-Ketis Z, Kersnik J. Using movies to teach professionalism to medical students. BMC Med Educ 2011;11(60). [Capturado 25 maio 2017]. Disponível em: https:/ /www. ncbi.nlm.nih.gov/pmc/articles/PMC3180297

44. de la Croix A, Rose C, Wildig E, Willson, S. Arts-based learning in medical education: the students' perspective. Med Educ 2011; 45(11):1090-1100.

45. Housen A. An esthetic thought, critical thinking and transfer. Arts Learn J 2002;18(1):2001-2002.

46. Reilly JM, Ring J, Duke L. Visual thinking strategies: a new role for art in medical education. Fam Med 2005;37(4):25052.

47. Osman M, Eacott B, Willson S. Arts-based interventions in healthcare education. Med Humanit 2018;44(1):28-33. 
48. Elder NC, Goldenhar L. The art of observation: impact of a family medicine and art museum partnership on student education. 2006 Jun;38(6):393-98.

\section{CONTRIBUIÇÃO DOS AUTORES}

Lúcia Trindade da Silva Mairot participou do delineamento da pesquisa, da coleta de dados, da análise dos dados e da redação do artigo. Beatriz Biasi Gin da Costa participou da coleta. Thaís Pedrosa Moraes Heringer participou da coleta. Raquel Camargos Borges participou da coleta e análise de dados. Eliane Perlatto Moura participou do delineamento da pesquisa, da coleta de dados, da análise dos dados, da redação e revisão do artigo.

\section{CONFLITO DE INTERESSES}

Os autores declaram não haver conflito de interesses neste estudo.

\section{ENDEREÇO PARA CORRESPONDÊNCIA:}

Eliane Perlatto Moura

Rua Tomé de Souza 1322 apto 1201

Funcionários - Belo Horizonte - MG

CEP: 30140130

E-mail: elianeperlatto@gmail.com 